\title{
QUOTIENTS OF COUNTABLE COMPLETE METRIC SPACES
}

\author{
E. MICHAEL
}

The purpose of this note is to prove Theorem 1, and to indicate some generalizations. Theorem 1 provides an alternative approach to the result (see [6, Corollary 1.2]) that the rationals are a quotient image of the irrationals. ${ }^{1}$

THEOREM 1. Every countable metric space $Y$ is a quotient image of a countable complete metric space $X$.

Proof. For each $y \in Y$, let $X_{y}$ be $Y$ with $Y-\{y\}$ made discrete (i.e., $X_{y}$ is the set $Y$, topologized by calling open all sets $U \cup S$ with $U$ open in $Y$ and $S \subset Y-\{y\}$ ). Let $X=\sum_{y \in Y} X_{y}$ (the topological sum of the $X_{y}$ ), and let $f: X \rightarrow Y$ be the obvious map. ${ }^{2}$ The definitions imply that $f$ is quotient. Clearly $X$ is countable. To show that $X$ is completely metrizable ${ }^{3}$, it suffices to show that each $X_{y}$ has this property. Now $X_{y}$ is a regular space with a countable base, and is therefore metrizable. Moreover, $X_{y}$ is the union of the two discrete-hence absolute $G_{\delta}$-subsets $\{y\}$ and $X_{y}-\{y\}$, so $X_{y}$ is also an absolute $G_{\delta}$ and therefore completely metrizable. That completes the proof.

REMARK 1 . The conclusion of Theorem 1 remains valid if $Y$ is only assumed to be a countable space with a countable base. (In fact, the proof of [4, Lemma 4.2] implies that such a $Y$ is a continuous open-hence quotient-image of a countable Hausdorff space $Y^{\prime}$ with countable base, and the proof of Theorem 1 goes through to show that such a $Y^{\prime}$ is the quotient image of a countable complete metric space.)

Our next remark will be applied in [5].

REMARK 2. Theorem 1 can be generalized as follows: Suppose $Y$ is a firstcountable, regular space with a countable, closed subset $A$ for which $Y-A$ is completely metrizable. Then $Y$ is a countable-to-one ${ }^{4}$ quotient image of a complete metric space.

Proof. For each $y \in A$, let $X_{y}$ be $Y$ with $A-\{y\}$ made discrete, and let $X=\sum_{y \in A} X_{y}$. Then each $X_{y}$ is metrizable by the Nagata-Smirnov theorem; the rest of the proof is essentially the same as for Theorem 1 .

Received by the editors December 7, 1975.

AMS (MOS) subject classifications (1970). Primary 54B15, 54E50.

${ }^{1}$ This result follows from Theorem 1 because every nonempty, complete, separable metric space is a continuous open-hence quotient-image of the irrationals [1, Corollary 4.7].

2 This construction has also been used, in different contexts, by J. Nagata [7] and E. von Douwen [2].

${ }^{3}$ I.e., metrizable by a complete metric.

4 Without this requirement, the result is trivial, since every first-countable space is a quotient image of the topological sum of its convergent sequences.

- American Mathematical Society 1976 
Remark 3. The quotient maps in Theorem 1 and Remarks 1 and 2 are automatically biquotient in the sense of [3]. (This follows from [3, Proposition 3.3(c)].)

\section{REFERENCES}

1. A. V. Arhangel'skiī, Open and close-to-open mappings. Relations between spaces, Trudy Moskov. Mat. Obšč. 15 (1966), 181-223 = Trans. Moscow Math. Soc. 15 (1966), 204-250. MR 34 \#6725.

2. E. K. van Douwen, Nonstratifiable regular quotients of separable stratifiable spaces, Proc. Amer. Math. Soc. 52 (1975), 457-460.

3. E. A. Michael, Bi-quotient maps and Cartesian products of quotient maps, Ann. Inst. Fourier (Grenoble) 18 (1968), fasc. 2, 287-302, (1969). MR 39 \#6277.

4. _- On representing spaces as images of metrizable and related spaces, General Topology and Appl. 1 (1971), 329-343. MR 45 \#2681.

5. Complete spaces and tri-quotient maps (to appear).

6. E. A. Michael and A. H. Stone, Quotients of the space of irrationals, Pacific J. Math. 28 (1969), 629-633. MR 41 \# 1002.

7. J. Nagata, Quotient and bi-quotient spaces of M-spaces, Proc. Japan Acad. 45 (1969), 25-29. MR 39 \#6278.

Department of Mathematics, University of Washington, Seattle, Washington 98195 